Disponible en www.hormigonyacero.com

0439-5689 / Hormigón y Acero 2022; 73(298):25-34

https://doi.org/10.33586/hya.2020.2290

\title{
Ampliación del puente de Rande. Proceso de montaje del nuevo tablero
}

\section{Rande Bridge Expansion. Erection Process of the New Deck}

\section{Héctor Bernardo Gutiérrez ${ }^{*}$, , Juan José Consuegra Pérez ${ }^{\mathrm{b}}$, Felipe Tarquis Alfonso ${ }^{\mathrm{a}}$, Conchita Lucas Serranoc ${ }^{c}$, Fátima Otero Vieitez ${ }^{\mathrm{d}}$}

\author{
${ }^{a}$ Dr. Ingeniero de Caminos, Canales y Puertos. gGRAVITY S.A., Madrid, España \\ ${ }^{b}$ Ingeniero de Caminos, Canales y Puertos. Vias y Construcciones S.A., Madrid, España \\ ' Ingeniera de Caminos, Canales y Puertos. Jefa del Servicio de Estructuras, Geotécnica \\ y Obras Maritimas. gGRAVITY S.A., Madrid, España \\ ${ }^{d}$ Ingeniero de Caminos, Canales y Puertos. Directora General de Grandes Proyectos y Servicios Técnicos. Grupo Puentes
}

Recibido el 31 de mayo de 2019; aceptado el 19 de junio de 2020

\section{RESUMEN}

El puente de Rande es un punto de cruce esencial en la zona de la ría de Vigo. Durante los trabajos para su ampliación era imprescindible que todos los trabajos necesarios para construir la misma no interrumpieran el tráfico ni afectasen el entorno marino del estrecho de Rande. Por esta razón, el método de construcción del nuevo tablero tuvo por objeto minimizar las restricciones de tráfico manteniendo siempre un carril abierto en cada sentido y el no afectar el entorno, cumpliendo con la limitaciones impuestas por el puente existente. Esta comunicación presenta los detalles del método empleado a fin de lograr cumplir con estas exigencias a la par que conseguir un plazo de obra adecuado.

@2022 Hormigón y Acero, la revista de la Asociación Española de Ingeniería Estructural (ACHE). Publicado por Cinter Divulgación Técnica S.L. Este es un artículo de acceso abierto distribuido bajo los términos de la licencia de uso Creative Commons (CC BY-NC-ND 4.0)

PALABRAS CLAVE: Puente, atirantado, Rande, ampliación, proceso constructivo.

\section{ABSTRACT}

The Rande Bridge is an essential crossing point in the area of the Vigo Estuary. During the expansion works carried out it was essential that all the needed works did not affect neither the passing traffic nor the marine environment of the Rande Strait. For this reason, the construction method selected for the erection of the new deck was double aimed to minimizing traffic restrictions, always maintaining at least one lane open in each direction, and to not affecting the surrounding environment. These objectives were also conditioned by the limitations imposed by the existing bridge. This paper presents the details of the erection method selected to meet both these requirements and a proper schedule.

(O2022 Hormigón y Acero, the journal of the Spanish Association of Structural Engineering (ACHE). Published by Cinter Divulgación Técnica S.L. This is an open-access article distributed under the terms of the Creative Commons (CC BY-NC-ND 4.0) License

KEYWORDS: Bridge, cable-stayed, Rande, expansion, erection process.

1.

INTRODUCCIÓN

La AP-9, o autopista del Atlántico, es una vía estratégica de comunicación que vertebra la comunidad autónoma de Galicia de norte a sur, sirviendo de nexo entre las ciudades de Vigo y Pontevedra. De todas sus estructuras, destaca, sin duda, el

* Persona de contacto / Corresponding author:

Correo-e / e-mail: hbernardog@ggravityeng.com (Héctor Bernardo Gutiérrez). puente de Rande, construido entre 1973 y 1977 y puesto en servicio al tráfico en febrero de 1981.

En el año 2006, coincidiendo con el $25^{\circ}$ aniversario de su entrada en servicio, la Intensidad Media Diaria (IMD) de vehículos que circulaban por el puente alcanzó los 47000 vehículos, cifra que continuó incrementándose y acercando esta in- 
fraestructura a su capacidad límite, con los problemas que ello conlleva en cuanto a la sensibilidad del tráfico a cualquier tipo de incidencia y su influencia en la fluidez de la circulación. A la vista de esta circunstancia, la concesionaria de la Autopista encargó un proyecto para la ampliación del puente pasando de 4 a 6 carriles de circulación, además de acometer una renovación integral de la misma para extender su vida útil más allá de la inicialmente prevista.

El proyecto de ampliación del puente de Rande consiste en adosar dos tableros adicionales a cada lado del existente. Estos nuevos tableros se debían colocar por la cara externa de los pilonos para evitar interferencias. Por tanto, no hay continuidad entre los carriles originales y los de la ampliación, excepto por las seis plataformas de emergencia y evacuación que conectan el tablero nuevo y el antiguo, y que están distribuidas a lo largo del vano principal.

Los nuevos tableros laterales cuentan con una anchura de $7.35 \mathrm{~m}$ y un canto de $2.40 \mathrm{~m}$. Están suspendidos de nuevos tirantes anclados a la coronación de los pilonos existentes reproduciendo el patrón de los tirantes originales. No obstante, los tableros de la ampliación también están conectados estructuralmente a la plataforma existente mediante celosías que terminan en una unión articulada. En términos generales, el nuevo esquema estructural intenta reproducir el original, pero con algunas peculiaridades resultantes del hecho de que los tableros nuevos y el original están conectados, por tanto, interactúan para crear un nuevo sistema estructural [1].

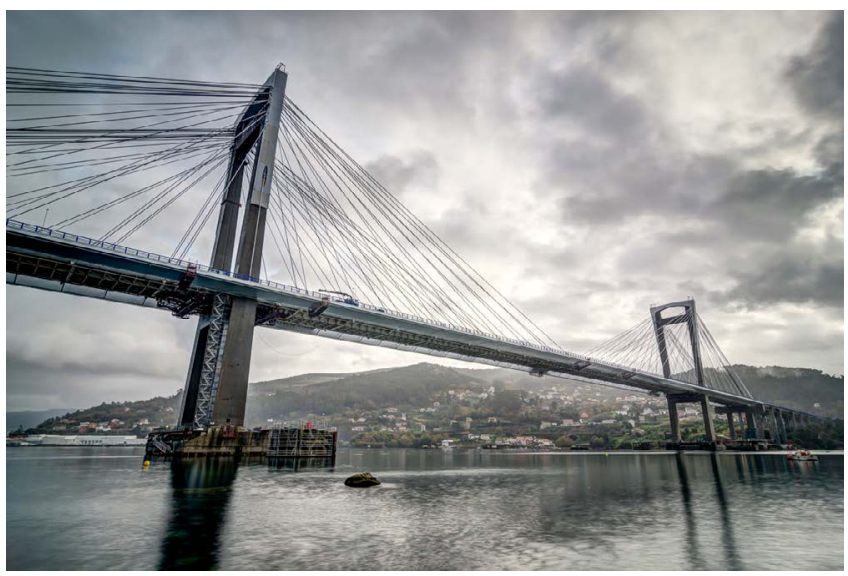

Figura 1. Vista del puente ampliado.

La ejecución de las actuaciones previstas en el proceso constructivo debía llevarse a cabo desde unas premisas fundamentales que, a la postre, han condicionado la construcción de la obra [2]:

- Una nula afección al tráfico rodado sobre el puente y sus accesos.

- El tráfico debía circular con la máxima seguridad durante los trabajos de ampliación.

- Nula afección medioambiental a la ría de Vigo.

- Máximo aprovechamiento de la capacidad resistente del puente actual.

- Plazo de ejecución extraordinariamente ajustado para una obra de estas características.

El presente trabajo aborda los trabajos de ampliación del tablero, siendo estos los últimos en realizarse una vez se hubie- ron ampliado las torres principales y las pilas estribo. Aquí nos limitaremos a la exposición del método constructivo elegido desde una perspectiva holística.

\section{2.}

\section{CONDICIONANTES PREVIOS DEL PROCESO DE MONTAJE}

A las premisas fundamentales citadas en el apartado anterior debían añadirse una serie de condicionantes adicionales, a saber:

- Necesidad de conectar la geometría de los nuevos tableros al existente con un número de puntos de unión de dovela superior al habitual.

- Imposibilidad de colocar medios de elevación pesados en el tablero existente.

- Incertidumbre sobre el estado tensional de los tirantes existentes.

- Imposibilidad de independizar el avance desde las dos torres por la presencia del tablero existente.

- Dificultad de colocación de las dovelas iniciales.

- Amplitud de los movimientos del nuevo tablero en construcción, superando los giros admisibles en los anclajes de los nuevos tirantes.

\section{3.}

\section{DEFINICIÓN DEL PROCESO DE MONTAJE}

\subsection{Aspectos previos}

El método de construcción más habitual en puentes atirantados es el conocido como avance en voladizo. Este método consiste en la construcción del tablero en porciones transversales, denominadas dovelas, apoyándose en la parte de puente ya construida. Habitualmente estas dovelas tienen una longitud igual a la distancia entre dos tirantes consecutivos y para su instalación se pueden usar métodos de ensamblaje en altura (como los empleados en la construcción del puente de Rande original) o de elevación de las dovelas completas una vez ensambladas en otras instalaciones. El segundo método tiene la ventaja de disminuir el número de operaciones de ensamble, soldadura y/o atornillado a realizar en altura, aumentando la productividad y la calidad del tablero final. Sin embargo, la magnitud y el tamaño de las piezas a izar requieren de medios de elevación específicos cuyo peso y coste supone una parte muy importante del total de la construcción. Adicionalmente, los esfuerzos introducidos en el tablero ya construido durante estas operaciones habitualmente requieren de refuerzos y análisis específicos más allá de los necesarios para garantizar el correcto funcionamiento del tablero como conductor de tráfico rodado.

El utilizar el propio tablero construido como apoyo para las nuevas partes también supone una complicación desde un punto de vista geométrico, pues la estructura sufre configuraciones de carga muy importantes en diferentes estados de construcción. Resulta complejo por tanto el conseguir la geometría final requerida y este aspecto es crucial durante la construcción, pues 
frecuentemente las dovelas colocadas han de situarse en posiciones ligeramente diferentes de las teóricas para poder acomodar errores de construcción, diferencias en el comportamiento de la estructura ya construida o las lógicas desviaciones asociadas a las tolerancias de fabricación y posicionamiento.

En el caso de la ampliación del puente de Rande no cabía otra solución distinta al avance en voladizo puesto que la estructura existente no era capaz de soportar el peso de los tableros de la ampliación sin la colaboración de los nuevos tirantes. Por tanto tan solo restaba definir el método de ensamblaje de cada dovela que conformaba un voladizo.

En este caso, la configuración en viga cajón elegida para el proyecto de la ampliación hacía que el ensamblaje y soldadura en altura por piezas fuese totalmente desaconsejable dada la gran cantidad de soldadura necesaria y la dificultad para garantizar un adecuado control geométrico. Por tanto, los $704.58 \mathrm{~m}$ de cada tablero de la ampliación se dividieron en dovelas transportables y manejables a fin de poder elevarlas a su posición definitiva. Se definieron dovelas tipo de $21.06 \mathrm{~m}$ más una serie de unidades especiales correspondientes a las iniciales y a las de cierre.

Como ya se ha detallado en otros trabajos [2, 3], el hormigonado de la losa de hormigón se realiza tras haber procedido a la instalación y tesado inicial de los tirantes. La decisión de realizar este hormigonado en cada dovela y no esperar al final del montaje como estaba previsto inicialmente tenía un doble motivo: por un lado poder proveer una superficie de trabajo por detrás del frente de avance, aspecto muy importante pues recordemos que no se podía hacer uso del tablero existente. Por otro, el volumen de hormigón usado en cada hormigonado se redujo significativamente, minimizando las restricciones de tráfico a tan solo unas horas durante la noche dando cumplimiento a una de las premisas iniciales.

\subsection{Diseño conceptual de los elementos auxiliares de izado}

Elegido el método del avance en voladizo con izado de dovelas completas se presentaba un problema que tampoco se encuentra habitualmente en la construcción de puentes atirantados de nueva factura y que es dónde situar el elemento auxiliar de izado de las cargas. En un puente de nueva construcción, y de no usarse medios de elevación externos tales como grúas, el dispositivo elevador de cargas, habitualmente conocido como carro de izado, solo puede situarse en el extremo construido del voladizo en ejecución. En el caso del puente de Rande, además de este voladizo se tiene el tablero existente, el cual proporciona una plataforma inmejorable para situar un elemento de izado. Sin embargo las innegables ventajas de tener una plataforma cercana se ven afectadas de los no pocos condicionantes que dicho tablero pone a la construcción.

En primer lugar la capacidad del tablero existente es limitada. No olvidemos que en todo momento se debía mantener el tráfico en el puente, por lo que parte de su capacidad está reservada a la carga de uso. Además, su estructura no se diseñó pensando en una futura ampliación, por tanto la resistencia de sus elementos es relativamente limitada. Un carro de elevación suspendido únicamente del tablero existente, si bien técnicamente posible, era una opción que hubiese requerido de refuerzos de su estructura dado lo elevado del peso del propio carro. En segundo lugar, el hecho de cargar el tablero con un peso tan elevado como el de una dovela del nuevo tablero, induce una deformación del puente existente que inevitablemente se transmite también a la nueva estructura, que se encuentra conectada por medio de las rótulas de unión. En otras palabras, el comportamiento de la nueva estructura se ve afectado por el de la existente y viceversa, lo cual introduce una variable adicional a la hora de predecir y controlar la geometría del nuevo tablero, factor muy importante en puentes de nueva construcción [4] pero que se vuelve fundamental a la hora de replicar una geometría ya existente.

Por otro lado, la opción de izar las nuevas dovelas utilizando para ello un carro apoyado exclusivamente en la parte construida de la ampliación también tenía una serie de desventajas que desaconsejaban dicha opción. La particular configuración del atirantamiento de la ampliación, dejando la plataforma de rodadura a modo de voladizo y descargando por tanto los tirantes existentes, si bien presenta ventajas para la estructura existente, lleva implícita una gran flexibilidad transversal del tablero de la ampliación. El introducir el peso de un carro de izado mas el peso de la propia dovela, con un radio de izado superior a los $10 \mathrm{~m}$, suponía un giro y descenso inaceptables geométricamente para el funcionamiento de la maquinaria de elevación. Asimismo magnificaba enormemente la problemática relacionada con el control de la geometría. Adicionalmente, la estructura de la ampliación debería haberse reforzado convenientemente para aceptar las cargas de construcción.

Por estos motivos se decidió escoger una opción intermedia entre la de izar apoyándose exclusivamente en el tablero original o la de hacerlo limitándose exclusivamente al nuevo. Dicha opción utilizaba dos carros de izado que compartían el peso de la dovela a izar repartiéndolo entre las dos estructuras. El primero de ellos, llamado carro trasero, se situaba en el frente de la última dovela construida del tablero de la ampliación, en la misma manera de un carro tradicional, con la salvedad de un reducido peso y complejidad, habida cuenta de que solo debía izar aproximadamente la mitad del peso de una dovela tipo. El segundo, llamado carro delantero, se situaba anclado bajo el tablero existente, y era un carro compartido para ambos lados de la ampliación. Su estructura era más complicada que la de los carros traseros, al igual que su peso, pero su posicionamiento en el lugar adecuado permitía maximizar la capacidad resistente del tablero existente a la par que izar dos dovelas al mismo tiempo, una de cada lado.

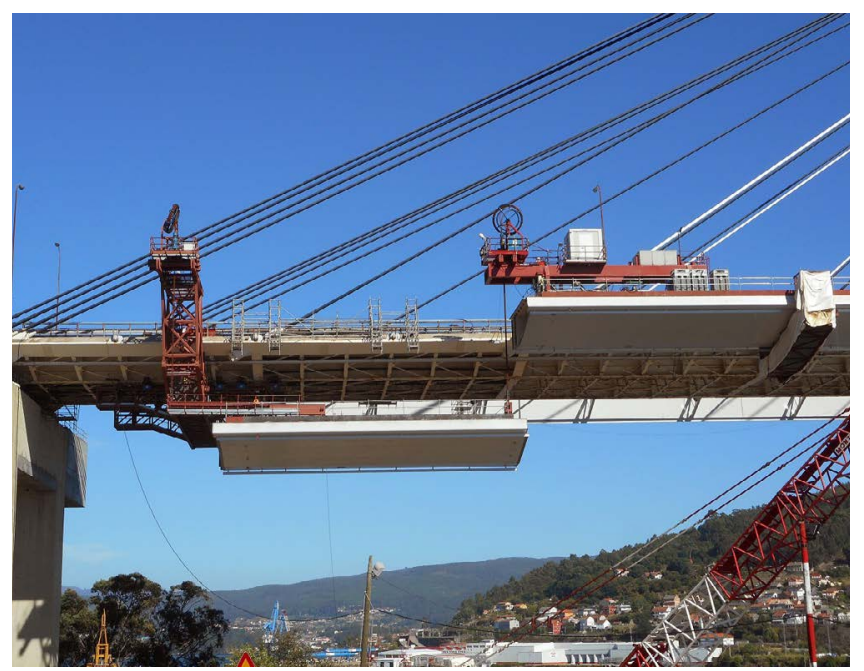

Figura 2. Izado compartido entre los dos carros. 


\subsection{Fases constructivas}

El proceso final elegido constaba de las siguientes fases:

- Carga y transporte de dovela a izar.

- Izado por medio de carros auxiliares de izado.

- Soldadura parcial de la dovela.

- Conexión de las rótulas al tablero existente.

- Liberación de los carros de izado.

- Instalación y tesado inicial del tirante.

- Instalación de la ferralla y hormigonado de la losa superior.

- Avance de los carros de izado.

- Finalización de la soldadura.

4.

\section{ELEMENTOS AUXILIARES DE MONTAJE}

\subsection{Dovelas iniciales}

Una vez elegido el método de construcción para las dovelas tipo y los medios auxiliares necesarios restaba planificar la instalación de algunas dovelas singulares, como eran las iniciales y las finales, es decir, las que dan comienzo a la construcción del puente, permitiendo la instalación de los carros de izado, y las que proveen de continuidad al tablero uniendo los diferentes tramos en que se divide para su construcción.

Las primeras, llamadas dovelas cero, serían la plataforma de arranque para el resto de medios auxiliares. Se trata muy habitualmente de dovelas singulares en cuanto a configuración y tamaño. En el caso del puente de Rande, la falta de vinculación entre las torres y el tablero evita diferencias significativas entre estas dovelas y el resto. Sin embargo, esa misma falta de vinculación obligaba a instalar un mínimo de tres tirantes para conformar una estructura estable por si misma, dando lugar a una dovela de $45 \mathrm{~m}$ de longitud.

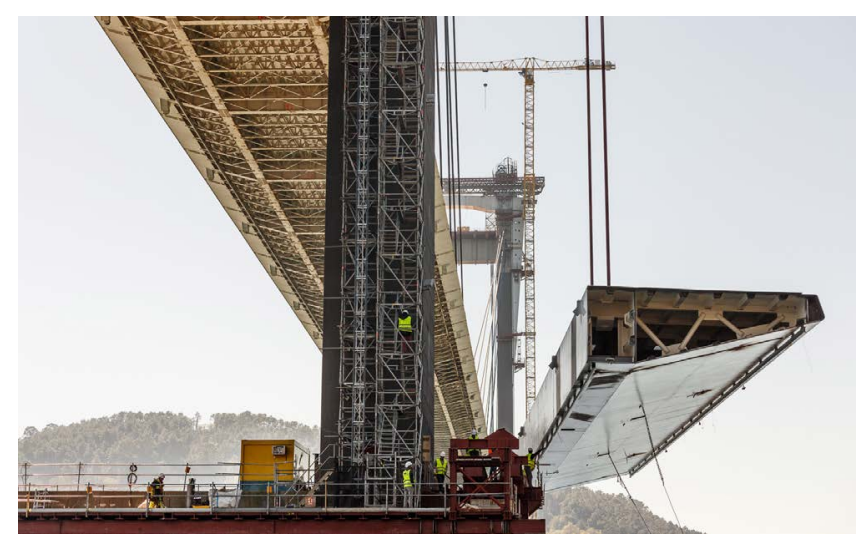

Figura 3. Izado de una dovela inicial.

Dado que en el momento de su colocación no existe tablero sobre el que colocar los carros de izado traseros y que, por motivos de peso, no era posible el izado con dos carros delanteros hubo de utilizarse un sistema independiente para la colocación de estas dovelas. Este sistema consistió en el mismo pórtico de izado situado sobre las torres de atirantamiento que ya fue utilizado para la ejecución de los trabajos del cabecero y descrito en [5]. Por capacidad estos pórticos eran capaces de levantar estas dovelas y mantenerlas suspendidas hasta que los tres tirantes requeridos eran instalados a una fuerza adecuada.

Dada la longitud de la pieza izada ( $45 \mathrm{~m}$ ), el escaso apoyo disponible (cara exterior de las torres) y lo inestable de la configuración, se hizo necesario disponer de un sistema de guiado que permitiesen mantener la posición en planta de las piezas durante su izado, de manera que no se produjesen giros ni desplazamientos incontrolados. Esta circunstancia era especialmente importante en el lado donde se ubicaban las grúas torre auxiliares, pues las dovelas tenían un hueco que permitía el paso del fuste de la grúa a fin de poder mantener esta operativa hasta el final del proceso cuando, tras su retirada, se procedía al cierre de dicho hueco. Este sistema de guiado de la posición consistía en una combinación de apoyos en el paramento de las torres y un sistema de dos cables cruzados que inducían la compresión de la dovela contra la cara de la pila a la vez que impedían su giro de eje vertical.

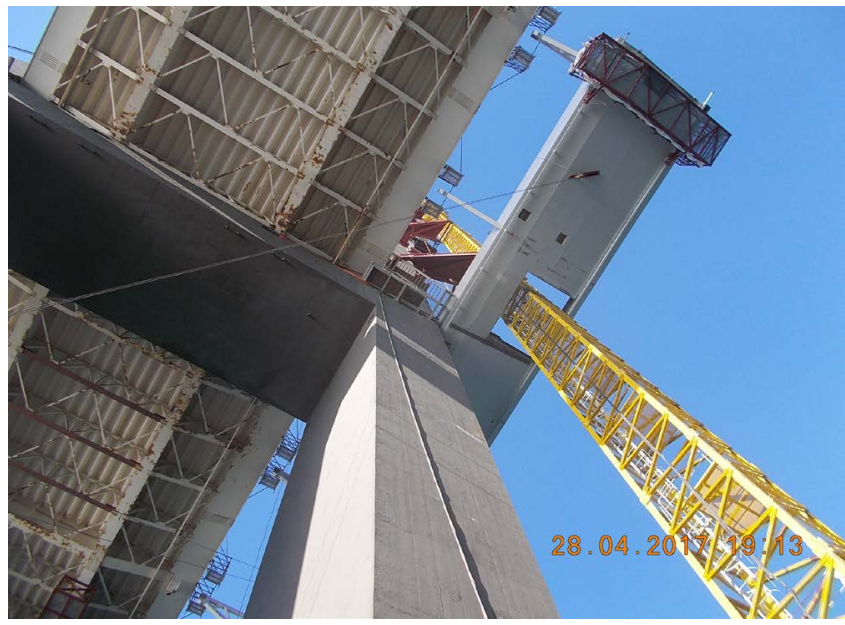

Figura 4. Hueco en dovela inicial para paso de grúa torre.

Los dispositivos de guiado de apoyo en la pila consistían en unos soportes metálicos con ruedas de apoyo que permitían su deslizamiento sobre la superficie del hormigón. El contacto se garantizaba por el propio peso de la dovela y por la componente horizontal del tiro de los cables de arriostramiento. Sin embargo, en las caras frontal y trasera de los pilonos la reacción se proporcionaba por medio de un dispositivo hidráulico que se encargaba de mantener una fuerza constante entre las ruedas y el paramento a la vez que resultaba en un par de fuerzas autoequilibrado y permitía adaptarse a la variación del paramento, originada por las dimensiones variables de la sección transversal de las torres.

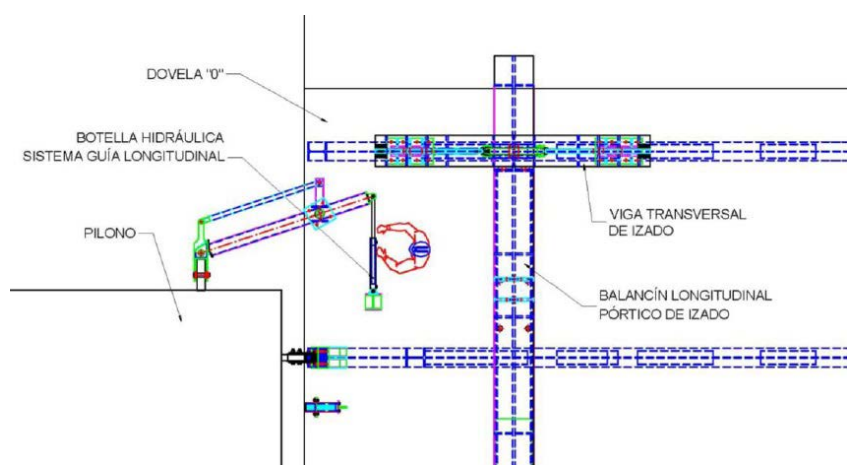

Figura 5. Sistema de guiado longitudinal adaptable a la pila. 
Por su parte, el sistema de cables instalado se configuró en planta formando una cruz a $40^{\circ}$, mientras que su ángulo y longitud variaban a medida que la posición vertical de la dovela cambiaba. Para poder proveer una tensión constante y equilibrada entre los dos cables se desarrolló un sistema en configuración de reenvío simple, con un extremo situado en unos cabrestantes fijados a la cimentación y el otro en un dispositivo hidráulico de control de tensión que aseguraba que los cabestrantes siempre proporcionaban la misma carga y que ambas estaban equilibradas para no introducir giros en la dovela. La baja velocidad ascensional de la maniobra ayudaba al funcionamiento sincronizado del sistema.

Por último, las dovelas se suspendían por medio de un balancín de izado con posibilidad de ajuste de la posición en el sentido transversal para ajustarse a la incertidumbre lógica del centro de gravedad de la dovela, de manera que se eliminasen los giros producidos por esta circunstancia.

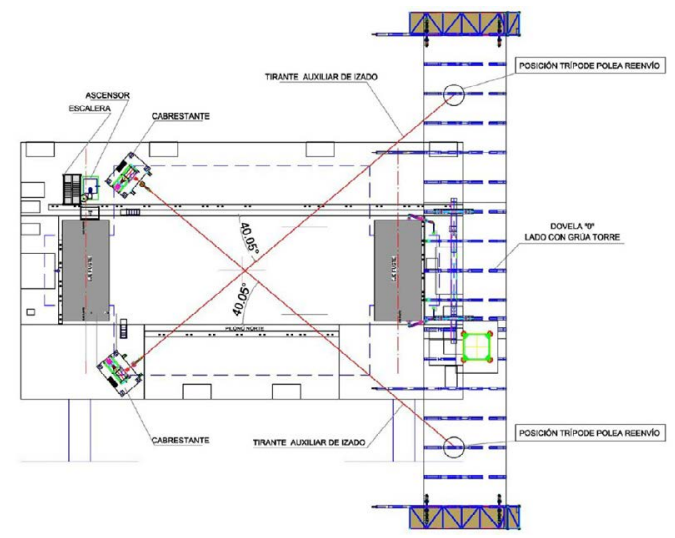

Figura 6. Sistema de guiado y arriostramiento transversal por medio de cabrestantes.

\subsection{Dovelas tipo}

Las dovelas tipo comprendian el mayor número de todas las instaladas y requirieron el diseño y fabricación de 4 unidades de carro delantero ( 1 por frente) y 8 trasero ( 2 por frente).

\subsubsection{Carro inferior delantero}

El diseño del carro delantero se veía condicionado por la limitación a su peso impuesta por el tablero existente (100 toneladas) y por la necesidad de ser único para los dos lados de la ampliación. Se diseñó como una estructura en celosía consistente en dos cerchas de $30 \mathrm{~m}$ de longitud total separadas entre ellas $2.40 \mathrm{~m}$. Las celosías estaban fabricadas con perfiles HEB y configuradas en módulos conectados por uniones atornilladas de manera que fuese sencillo su suministro y montaje en obra.

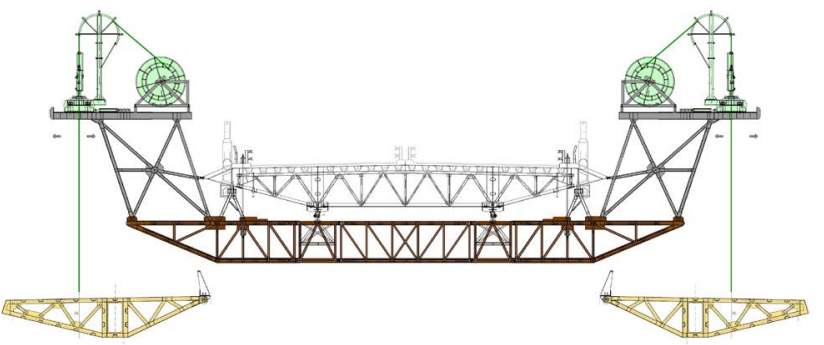

Figura 7. Carro inferior delantero.
Sobre los extremos de las cerchas se sitúan dos estructuras en torre y voladizo que permitían acoger en ellas los gatos de izado en la vertical de la dovela a elevar y a una altura adecuada para permitir la elevación a la cota final.

Durante su movimiento el carro se desplazaba gracias a un sistema de rodadura formado por cuatro ruedas que se conectaba a la platabanda inferior de las vigas principales del tablero existente y sobre las que se desplazaba una viga carril. Según avanzaba el carro, unos operarios se encargaban de mover las ruedas ancladas a la platabanda por delante del carro. Durante la maniobra de izado de dovelas sin embargo, el carro se suspendía de unas barras de alta resistencia pasantes al tablero y ancladas a yugos situados sobre la losa superior en la zona de hormigón de la viga de arcén, de manera que no se transmitía la carga a la platabanda inferior más cercana a la dovela izada sino prácticamente al tirante. En el lado contrario, la reacción contra dicha platabanda equilibraba el sistema de cargas verticales.

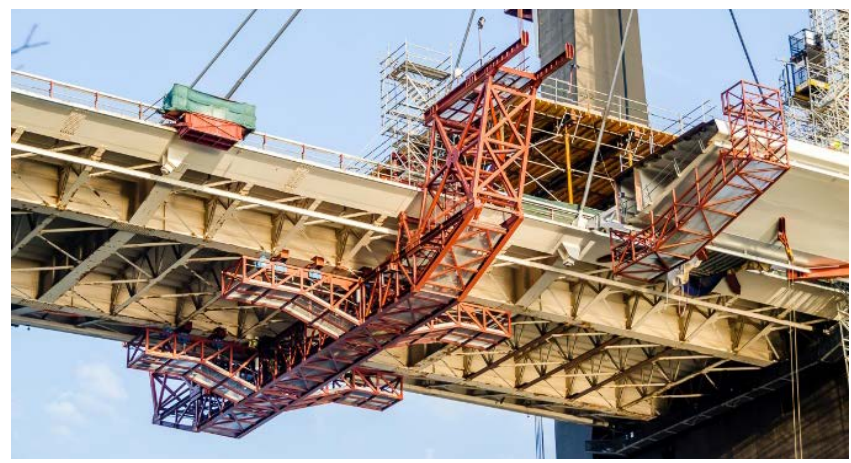

Figura 8. Carro inferior delantero.

Dado que la posición de la dovela izada chocaba contra la estructura del propio carro en el caso de compartir la misma alineación se hubo de diseñar una viga de izado en voladizo que permitiese desfasar la posición longitudinal de la dovela y la del carro. Además, un balancín se encargaba de ajustar la rotación de la dovela debido a la incertidumbre en la posición del centro de gravedad, al igual que ocurría en las dovelas iniciales.

\subsubsection{Carro superior trasero}

Los condicionantes de diseño del carro trasero no eran tan exigentes como los del delantero. La limitación de peso no era tan estricta y dado que cada tablero de la ampliación se encuentra a un lado de la torre, se habían de diseñar estructuras únicas, disminuyendo la complejidad del mecanismo.

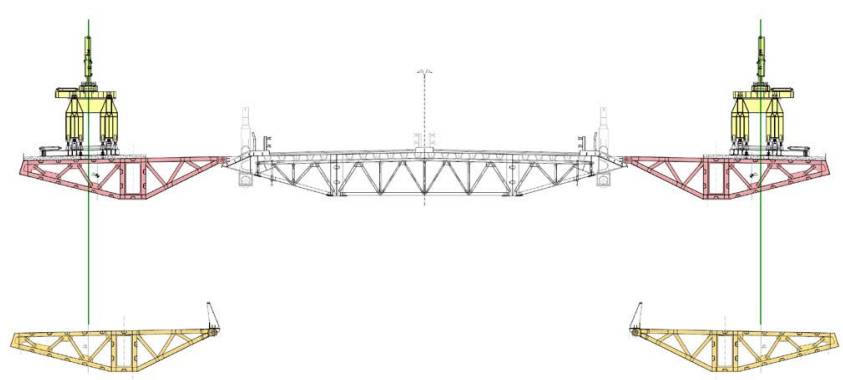

Figura 9. Carros superiores traseros.

La estructura del carro consistía en unas vigas principales encargadas de unirse a la dovela anterior y volar por encima de la 
dovela a izar permitiendo la colocación sobre ellas de los dispositivos de izado. En el voladizo frontal se situaba una segunda estructura denominada pieza de izado que permitía un ajuste tanto en longitudinal como en transversal a las vigas principales, de manera que se pudiese ajustar la posición de la dovela.

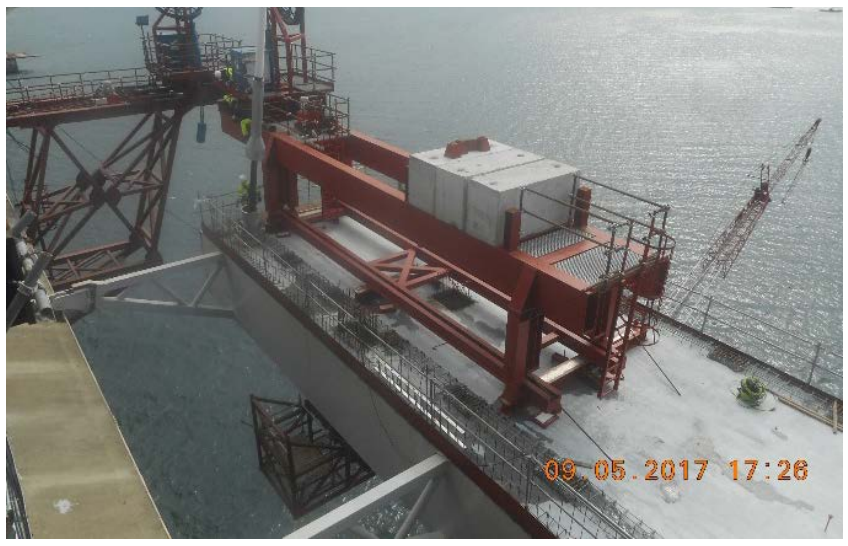

Figura 10. Carro superior trasero.

El apoyo de las vigas principales del carro se materializaba contra el hormigón del tablero de la ampliación por medio de vigas de apoyo. En el más trasero de los dos se disponía además de un contrapeso a base de bloques de hormigón destinado a compensar el tiro de la dovela izada a fin de equilibrar el vuelco del carro. Ambos apoyos permitían el avance de unas vigas carrileras sobre las cuales se avanzaba el carro a la dovela siguiente con posterioridad a la finalización de los trabajos de hormigonado de la losa.

\subsection{Dovelas de extremo}

Las dovelas a colocar en ambos extremos de los tableros de la ampliación eran singulares por varios motivos. En primer lugar por su propia geometría, que difiere de la dovela tipo en longitud y peso, ya que varios tirantes se anclan en un espacio reducido y a la vez han de acoger los péndulos de retenida que las unen a la pila estribo. En segundo lugar, no es posible elevarlas de manera puramente vertical ya que la última pieza ha de situarse sobre la pila estribo, siendo por tanto necesaria una operación de ripado longitudinal.

Recordemos, además, que el carro delantero de izado ha de situarse por delante de la dovela a izar, circunstancia que no es físicamente posible en este caso ya que chocaría con la pila estribo. A esto hay que sumarle que no era posible acceder con los medios habituales (pontona) a la vertical de elevación desde la parte inferior del puente en el lado norte, al estar en tierra firme.

En el caso de la Pila de Estribo Norte el problema se solucionó mediante el empleo de una grúa de gran tonelaje que compartía el izado con los carros traseros. Sin embargo, en el lado sur fue necesario desarrollar una estructura auxiliar que permitiese colocar las diferentes piezas metálicas salvando las restricciones mencionadas. Esta estructura consistía en un carro de lanzamiento formado por dos vigas en celosía y un carro superior capaz de deslizar con las piezas suspendidas. Este carro se apoyaba en su extremo sur sobre el tablero de los viaductos de acceso y en el extremo norte en el propio tablero de la ampliación.

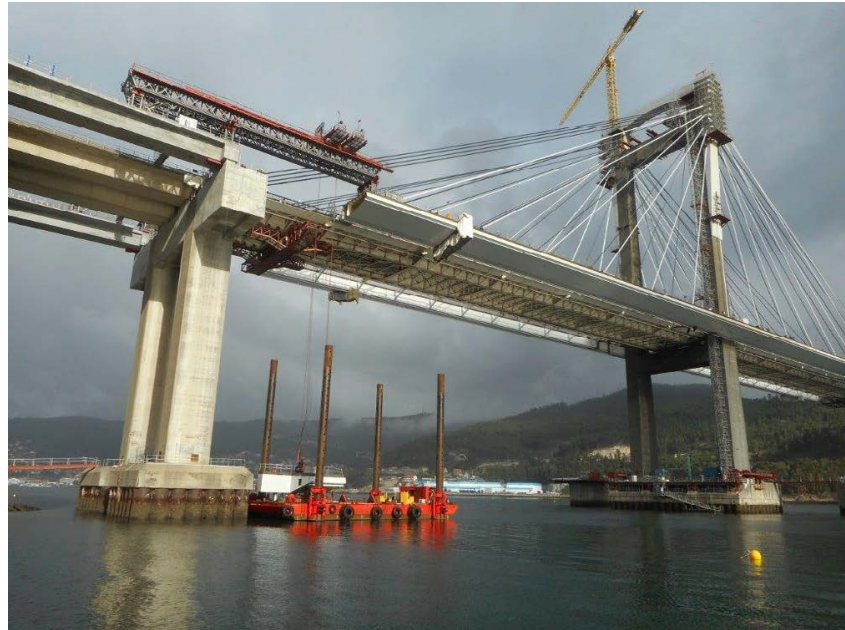

Figura 11. Viga de elevación de dovelas de extremo en el lado sur.

\subsection{Estructuras auxiliares}

Además de los elementos descritos destinados al izado de las dovelas, se diseñaron y fabricaron un total de 8 carros de soldadura que, suspendidos del tablero de la ampliación, permitían independizar el fin de los trabajos de soldadura y pintura de los carros de izado, proporcionando una superficie de trabajo protegida y segura para los operarios.

\section{5.}

\section{PROCESO CONSTRUCTIVO}

\subsection{Fabricación y transporte a obra de dovelas}

La fabricación de la estructura metálica es un punto clave de cuya precisión depende en gran medida el éxito de la construcción. Una adecuada planificación de la fabricación, con un proceso de montaje y soldadura adecuado unido a un control exhaustivo de la geometría son garantía de éxito en el montaje en obra. Asimismo, se hace necesario un montaje en blanco que garantice el casamiento de los bordes a unir en obra al igual que lo es disponer de las adecuadas zonas de ajuste destinadas a absorber los inevitables desvíos y errores encontrados durante la construcción.

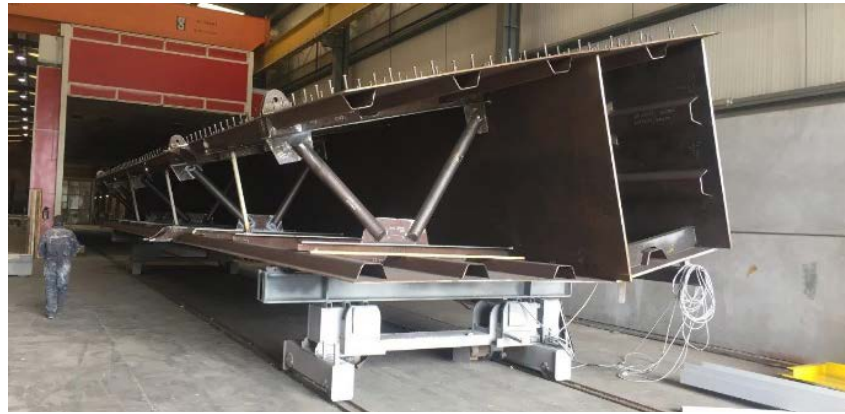

Figura 12. Fabricación en taller

Las dovelas tipo de $21.06 \mathrm{~m}$ de longitud fueron fabricadas en los talleres que las empresas Emesa y Tallers Dízmar tienen en A Coruña y en Carballiño respectivamente. 
Tras el corte y armado de la chapa se procedía a la soldadura, en todos los casos sometidos a una cuidadosa verificación geométrica, donde se verificaba el encaje del frente de cada dovela con la siguiente de manera que, en obra, se garantizase la alineación de los labios de soldadura. Para su transporte, y dependiendo del taller en cuestión, se acometía con la dovela completa salvo por las celosías de unión al tablero existente, o bien dividida longitudinalmente en dos mitades. En todos los casos las dovelas, una vez llegadas a la zona de obra acondicionada al efecto, seguían el proceso de completarlas con las celosías y elementos que quedasen pendientes de manera previa a su acopio y preparación para la carga en una pontona.

\subsection{Carga en pontona y transporte a posición de izado}

Una vez en obra, las dovelas debían transportarse a la zona de su izado, esto es, la vertical de los carros. Para ello se utilizó una pontona del tipo "Jack-up", esto es, autoelevable por medio de unas patas llamadas spuds. Esto permite independizarse del estado de la mar durante los trabajos ya que la plataforma conforma una superficie estable.

La carga de las dovelas en la misma se realizaba por medio de una grúa de gran tonelaje en un muelle de carga conocido como "El muelle de la piedra".

Una vez sobre la pontona, y realizado su trincaje, se procedía al remolque de la misma hasta la posición de izado donde por medio de anclas y cabrestantes se lograba la posición deseada que era fijada, alli donde el calado lo permitía, por medio de los spuds.

\subsection{Ensamblaje de dovelas iniciales}

Las dovelas iniciales que correspondían a los lados del tablero donde se situaban las grúas torres disponían de un hueco para el paso del fuste como ya se ha mencionado. Para poder materializar este paso era necesario completar el montaje de las dovelas alrededor de la propia grúa lo que imposibilitaba el suministro de los $45 \mathrm{~m}$ de dovela completos. Es por ello que estas dos dovelas en particular habían de suministrarse necesariamente en un mínimo de dos tramos que abrazasen la grúa y cuya soldadura debía realizarse bajo la posición definitiva.

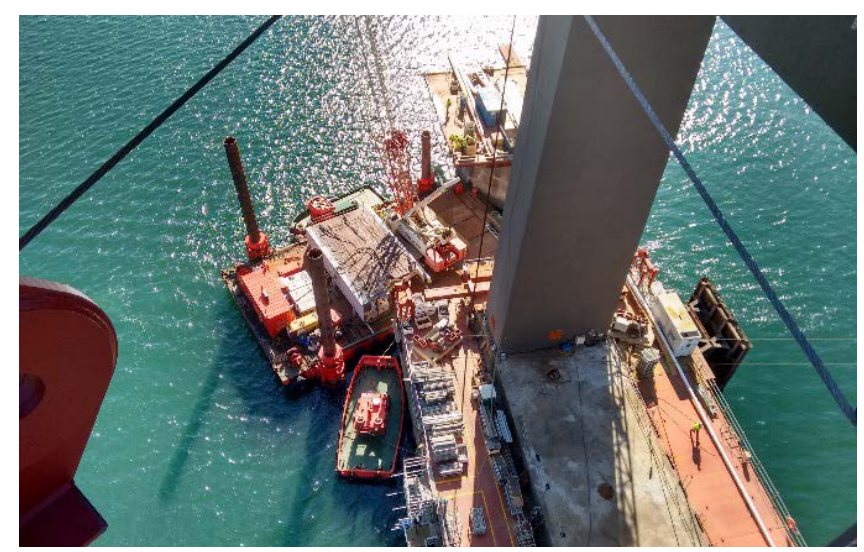

Figura 13. Ensamblaje de las dovelas iniciales en el lado grúa torre.

Finalmente se realizó una división en tres tramos que permitiese su descarga desde la pontona a las plataformas situadas en la base de las torres por medio de una grúa.

\subsection{Izado de dovelas}

Tras la conexión de los cables de izado a los balancines situados en la dovela se procedía a la toma de carga. Como se ha mencionado ya, la presencia de 4 spud en la pontona permitió aislar la maniobra de toma de carga del oleaje marítimo, facilitando enormemente el proceso y permitiendo el mismo con gran independencia de las condiciones de oleaje Una vez suspendida de ambos carros de izado la dovela, esta era elevada a su cota utilizando los gatos hidráulicos situados sobre los mismos. Un cuidado control geométrico permitía entonces colocarla en su posición teórica según los cálculos del proceso constructivo [3], momento en el que se comenzaba la soldadura de su extremo trasero a la última dovela soldada con anterioridad. Tras completar un cierto porcentaje de esta soldadura se podía proceder a la soldadura de las rótulas de unión al tablero antiguo.

\subsection{Conexión de las rótulas de unión al tablero existente}

Las rótulas de unión son las artífices del funcionamiento conjunto y compatibilidad de ambos tableros. Son elementos industriales que gracias a su esfericidad permiten la articulación en tres ejes mientras que restringen los movimientos de traslación. Dado que se debía garantizar una durabilidad suficiente, su ensamblaje se ha realizado en taller, limitándose las actuaciones en obra a la soldadura de unas chapas de gran espesor que las conectan a las ménsulas de celosía que unen tablero existente y ampliación. El diseño de esta conexión soldada ha permitido además, asumir las tolerancias y errores lógicos de toda fabricación metálica a esta escala.

\subsection{Instalación de los nuevos tirantes}

Unidas las rótulas y las caras de dovela, se procedía a la liberación de la carga en los carros de manera que el peso de la dovela estaba resistido únicamente por el tablero de la ampliación y el existente. En ese momento, se procedía a comenzar la instalación del tirante correspondiente a la dovela en cuestión, con un control completo de la carga en el mismo. Dicha instalación tenía el efecto de recuperar parte de la flecha y el giro ocurridos en la dovela al liberar su peso de los carros.

La instalación de los tirantes se realizó por control de carga, siempre asociado a un riguroso control de la posición del voladizo delantero. A fin de independizar estas mediciones de los movimientos del tablero existente (debidos a tráfico, viento y efectos térmicos) se realizó un estudio topográfico con medición continua del comportamiento del puente bajo las cargas de tráfico y efectos térmicos, de manera que se determinó previamente el momento más adecuado para efectuar las mediciones de control.

Las labores de instalación del atirantamiento se describen con detalle en otro trabajo [6]. Los tirantes se instalaron tesándolos como mínimo a $300 \mathrm{MPa}$. Esta tensión mínima era necesaria para garantizar el funcionamiento correcto de las cuñas de anclaje y evitar que el tirante pudiese deslizar. El proceso seguido, en el que los ajustes del tablero se han realizado al final del proceso ha permitido que no haya que realizar múltiples ajustes durante la construcción, ajustes que habitualmente llevan asociadas mordidas del gato de tesado en posiciones muy próximas, lo cual no es admisible. 


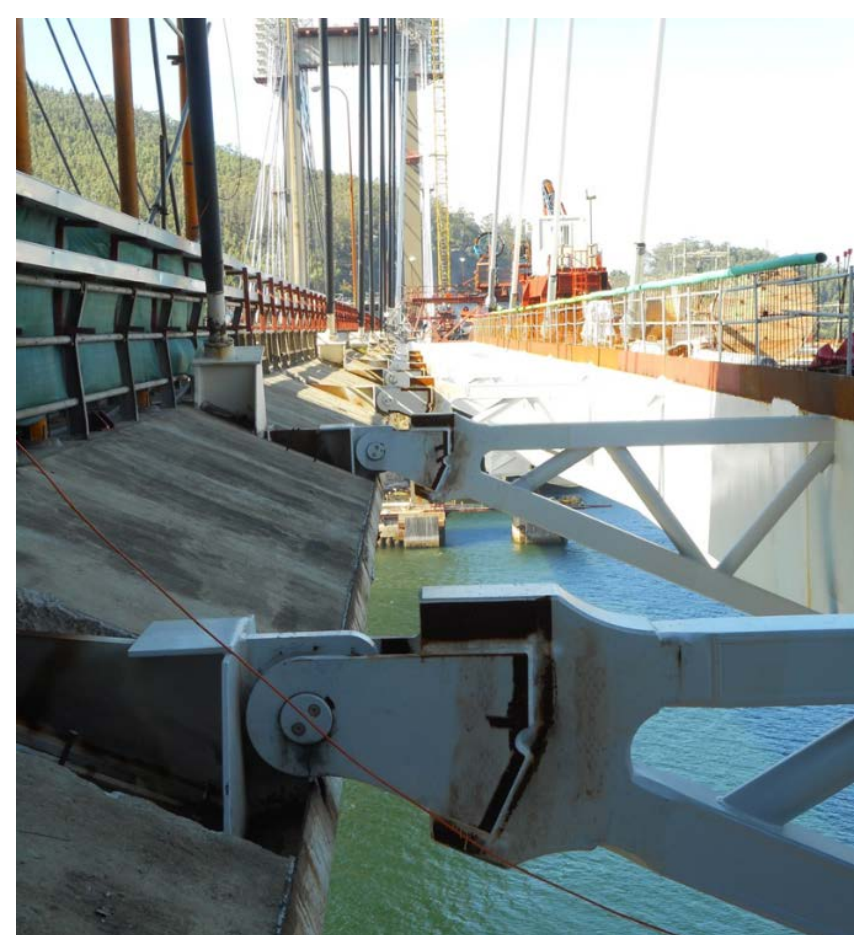

Figura 14. Detalle de union de la rótula y la dovela.

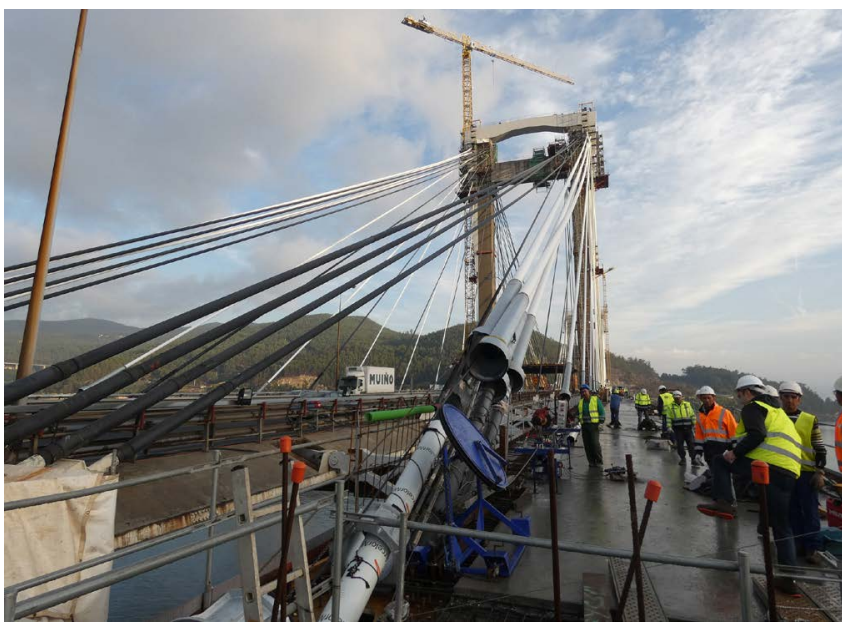

Figura 15. Trabajos de instalación de los tirantes.

\subsection{Ferrallado y hormigonado de la losa superior}

Con el tirante instalado y tesado a una cierta fuerza, se procedía al ferrallado y hormigonado de la losa de hormigón que completa la sección mixta. Esta labor se veía facilitada por la posibilidad de suministro de personal y materiales desde el tablero de la ampliación ya construido, que formaba una plataforma inmejorable de acceso.

El hormigonado de la losa se realizó siempre en horario nocturno con corte de un solo sentido de circulación, desviando el tráfico por los otros dos carriles del sentido contrario en las horas de menor densidad circulatoria.

\subsection{Avance de los carros a la siguiente dovela}

Una vez el hormigón de la losa alcanzaba la resistencia requerida y si la dovela del otro lado del tablero estaba completada, se procedía al movimiento de los carros traseros y delanteros. Hay que tener en cuenta que esta maniobra estaba encajada dentro de una secuencia que involucraba los 8 frentes de avance como se ha explicado en [3], por lo tanto no era completamente independiente.

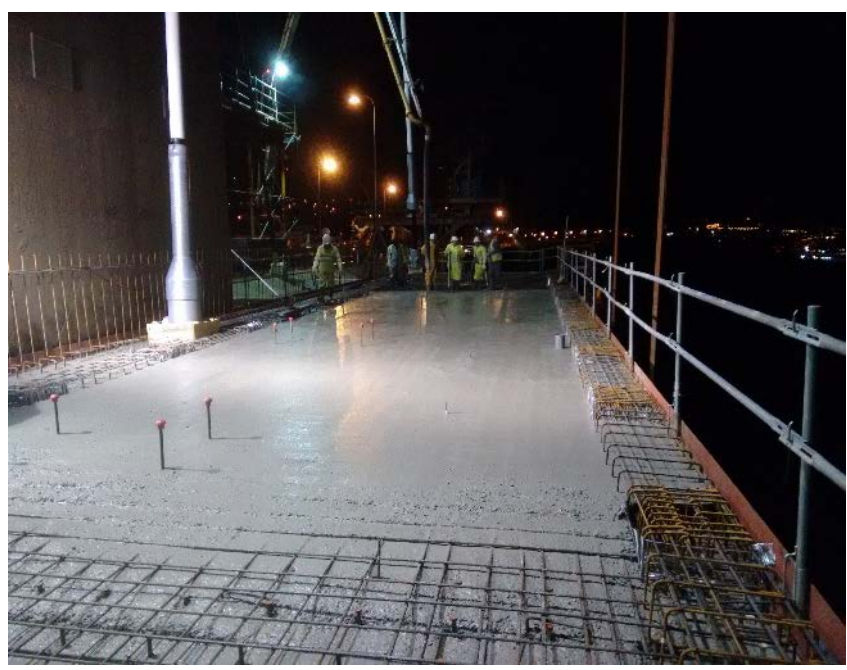

Figura 16. Trabajos nocturnos de hormigonado de la losa.

Finalizada esta fase se estaba en disposición de acometer una nueva fase de izado hasta completar el total del tablero hasta alcanzar las dovelas singulares de cierre en extremos y en centro de vano.

\subsection{Dovelas de cierre del vano central}

Las dovelas de cierre del vano central se izaron empleando solo los carros de izado traseros de cada lado tras la retirada de los delanteros. Se emplearon sistemas de bloqueo simples para evitar los movimientos diferenciales entre ambos lados de la dovela. No obstante, a diferencia de lo que suele ocurrir en la construcción de puentes atirantados nuevos, estos movimientos eran reducidos, puesto que las nuevas dovelas ya estaban unidas al puente original, por lo que sus movimientos se encontraban muy restringidos.

\subsection{Instalación de la carga muerta y retesado final.}

Una vez finalizado el izado e instalación de todas las dovelas, con sus correspondientes tirantes y el hormigonado de la losa, se procedió a la instalación de la carga muerta del tablero, correspondiente al pavimento y las barreras anti impacto. Finalizada esta instalación se hacía preceptivo un retesado de todos los tirantes del puente a fin de conseguir el estado de carga en los mismos y la geometría deseada.

\section{6. \\ CONCLUSIONES}

El proceso de construcción del tablero de la Ampliación del puente de Rande se ha llevado a cabo mediante una variación del proceso de avance en voladizo con izado de dovelas completas tradicional, consistente en el reparto del peso de la dovela izada entre dos carros de izado situados uno en el tablero 


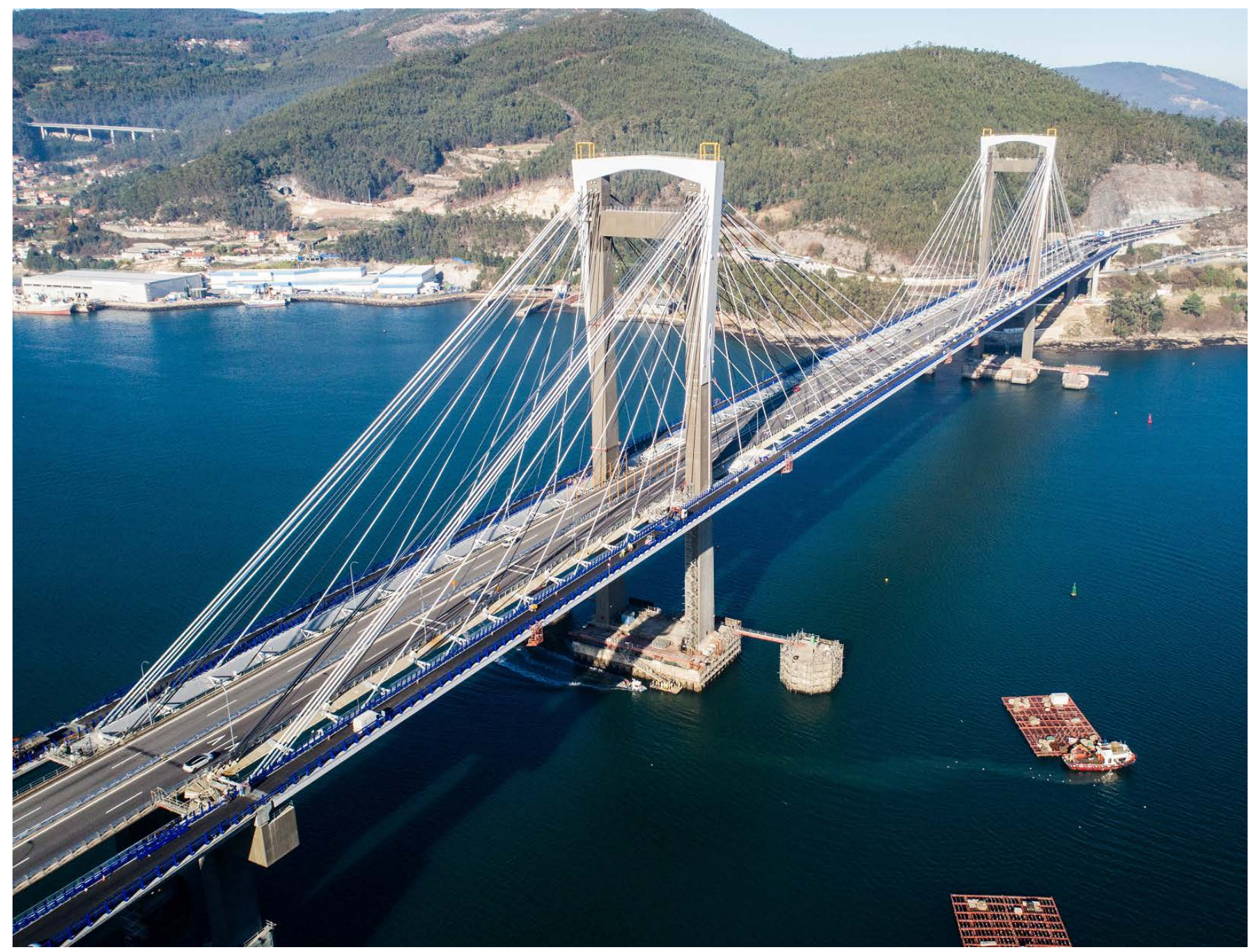

Figura 17. Vista del puente finalizado.

existente y otro en el tablero de la ampliación.

Esto junto con el diseño de los carros de izado, han presentado las siguientes ventajas:

- Se han minimizado los cambios geométricos debido a los carros de izado.

- Los trabajos de tesado y retesado de los tirantes se han reducido y se han evitado los problemas relacionados con la mordida de las cuñas entre dos posiciones cercanas.

- Se ha mejorado el control geométrico de la estructura.

- Se ha reducido el plazo de construcción total.

La eficacia del método seguido permitió completar la obra y abrir al tráfico en diciembre de 2017, cumpliendo con los plazos establecidos con el cliente y alcanzado un destacable periodo de construcción de tan solo 15 meses.

\section{Agradecimientos}

Los autores quieren agradecer a todas las partes implicadas en este proyecto su colaboración, imprescindible para llevarlo a buen término, en especial al Ministerio de Fomento, AUDASA,
Grupo Puentes, MC2 Estudio de Ingeniería, Julio Martínez Calzón, Manuel Juliá Vilardell FHECOR Ingenieros Constructores, Pondio Ingenieros y APIA XXI.

\section{Referencias}

[1] Serrano Corral, Á. \& Rupérez Astarloa, M. (2020) «Ampliación del puente de Rande. Planteamiento general y concepción del proyecto» de VIII Congreso de ACHE, Santander, 2020.

[2] Serrano Corral, Á \& Rupérez Astarloa, M. (2020) «Ampliación del puente de Rande. Principios generales y condicionantes del proceso de construcción» de VIII Congreso de ACHE, Santander, 2020.

[3] Corres Peiretti, H., De Cabo Ripoll, J., Sánchez Delgado, J., Tarquis Alfonso, F., Bernardo Gutiérrez, H. \& Arroyo Márquez, J. (2020) «Ampliación del puente de Rande. Diseño del proceso constructivo» de VIII Congreso de $A C H E$, Santander, 2020.

[4] Gozález-Valerio, J.A. \& Lucas Serrano, C. (2016) «Control geométrico del tramo atirantado del puente de la Constitución de 1812 sobre la Bahía de Cádiz» Hormigón y Acero, vol. 67, nº 278-279, pp. 143-158, 2016.

[5] Otero Vieitez, F. (2020) «Ampliación de los cabeceros de pilonos en la ampliación del puente de Rande,» de VIII Congreso de ACHE, Santander, 2020. 
[6] Sánchez de Prado, J., Del Espino Monedero, M., Vilchez Motino, P., Trotin, N. \& Gómez Gómez, Z. «Los tirantes de la ampliación del puente de Rande,» de VIII Congreso de ACHE, Santander, 2020.

[7] Bernardo, H., Tarquis, F., Lucas, C., \& Viartola, L.M. (2019). Rande Bridge Widening: A 400-m-Span Cable-Stayed Bridge Expansion. Practice Periodical on Structural Design and Construction, 24(4).

[8] Serrano-Corral, Á., Ruperez-Astarloa, M., Alonso-López, J.C., \& Rodríguez-Muñoz, D. (2019). Widening of the Cable-Stayed Bridge Over the Rande Strait in Spain. Structural Engineering International, 29(4), 547-550. 